\title{
Activation of MAPK pathways links LMNA mutations to cardiomyopathy in Emery-Dreifuss muscular dystrophy
}

Antoine Muchir, ${ }^{1}$ Paul Pavlidis, ${ }^{2}$ Valérie Decostre, ${ }^{3,4,5}$ Alan J. Herron, ${ }^{6}$ Takuro Arimura, ${ }^{3}$ Gisèle Bonne, ${ }^{3,4,5}$ and Howard J. Worman'

\begin{abstract}
'Department of Medicine and Department of Anatomy and Cell Biology and 2Department of Biomedical Informatics, College of Physicians and Surgeons, Columbia University, New York, New York, USA. ${ }^{3}$ INSERM U582, Institut de Myologie, Paris, France. ${ }^{4}$ Faculté de médecine, Université Pierre et Marie Curie, Paris 6, Paris, France. ${ }^{5}$ Assistance Publique-Hôpitaux de Paris, Groupe Hospitalier Pitié-Salpêtrière, Unité Fonctionelle Myogénétique et Cardiogénétique, Service de Biochimie B, Paris, France. ${ }^{6}$ Institute of Comparative Medicine and Department of Pathology, College of Physicians and Surgeons, Columbia University, New York, New York, USA.
\end{abstract}

\begin{abstract}
Mutations in LMNA, which encodes nuclear Lamins $\mathrm{A}$ and $\mathrm{C}$ cause diseases affecting various organs, including the heart. We have determined the effects of an Lmna H222P mutation on signaling pathways involved in the development of cardiomyopathy in a knockin mouse model of autosomal dominant Emery-Dreifuss muscular dystrophy. Analysis of genome-wide expression profiles in hearts using Affymetrix GeneChips showed statistically significant differences in expression of genes in the MAPK pathways at the incipience of the development of clinical disease. Using real-time PCR, we showed that activation of MAPK pathways preceded clinical signs or detectable molecular markers of cardiomyopathy. In heart tissue and isolated cardiomyocytes, there was activation of MAPK cascades and downstream targets, implicated previously in the pathogenesis of cardiomyopathy. Expression of H222P Lamin A in cultured cells activated MAPKs and downstream target genes. Activation of MAPK signaling by mutant A-type lamins could be a cornerstone in the development of heart disease in autosomal dominant Emery-Dreifuss muscular dystrophy.
\end{abstract}

\section{Introduction}

Dilated cardiomyopathy is the most serious aspect of Emery-Dreifuss muscular dystrophy (EDMD), which is also characterized by contractures of the elbows, Achilles' tendons, and spine and progressive muscle wasting in a humero-peroneal distribution (1). Initially described as an X-linked inherited disorder, autosomal dominant and recessive forms are also recognized, with the dominant form being most prevalent. X-linked EDMD arises as a consequence of mutations in emerin (EMD) (2), encoding the ubiquitously expressed inner nuclear membrane protein emerin $(3,4)$. Autosomal dominant and recessive EDMD arise from mutations in $\operatorname{LMNA}(5,6)$. Several other diseases affecting striated muscles or other tissues are also caused by different LMNA mutations (7).

LMNA encodes A-type nuclear lamins, of which Lamin A and Lamin C are the predominant somatic cell isoforms (8). Nuclear lamins are intermediate filament proteins that polymerize to form $10-\mathrm{nm}$ diameter filaments on the inside of the inner nuclear membrane (9-12). The lamina interacts with integral proteins in the inner nuclear membrane, provides anchorage sites for chromatin, and structurally supports the nuclear envelope (7). Many disease-causing A-type lamin mutants induce abnormal nuclear envelope architecture (7).

Although A-type lamins are expressed in most human tissues, EDMD selectively affects striated muscle and tendons. The reason for this tissue-selective phenotype is not clear, but 2 hypotheses have emerged to explain it (7). The mechanical stress hypothesis propos-

Nonstandard abbreviations used: ANF, atrial natriuretic factor; EDMD, Emery-Dreifuss muscular dystrophy; MHC, myosin heavy chain; MLC-2, myosin light chain 2 .

Conflict of interest: The authors have declared that no conflict of interest exists. Citation for this article: J. Clin. Invest. 117:1282-1293 (2007). doi:10.1172/JCI29042. es that mutations in A-type lamins lead to increased nuclear fragility and eventual nuclear disruption in tissues subjected to mechanical strain and is based primarily on observations in cultured cells. The gene expression hypothesis proposes that mutations in A-type lamins lead to abnormal tissue-specific gene regulation and is based on findings that A-type lamins and associated proteins bind to chromatin and transcriptional regulators. These 2 models are not mutually exclusive, as defective nuclear mechanics have been linked to altered expression of stress-activated genes in fibroblasts lacking A-type lamins (13). However, despite data obtained mostly from cultured cells and in vitro binding assays that have lead to the mechanical stress and gene expression hypotheses, there are scant experimental results linking mutations in A-type lamins to pathogenic pathways in affected tissues. Here we report results of an analysis, using hearts from a mouse model of EDMD, exploring effects of an A-type lamin mutation on gene expression and signaling pathways involved in development of cardiomyopathy.

\section{Results}

Gene expression profiling in hearts from mice with Lmna H222P mutation. To identify abnormal expression of genes involved in development of cardiomyopathy caused by Lmna mutation, we carried out a genome-wide RNA expression analysis in hearts from $L m n a^{H 222 P /+}$ and $L m n a^{H 222 P / H 222 P}$ mice. A detailed description of these mice has been previously published (14). Male $L m n a^{H 222 P / H 222 P}$ mice develop cardiac chamber dilation, decreased left ventricle fractional shortening and hypokinesis detectable by echocardiography at 8 weeks of age. At 12 weeks of age, conduction system abnormalities become pronounced, characterized primarily by an increased PR interval on electrocardiograms. Histological analysis shows left ventricular fibrosis, fiber degeneration, and atrial dilation by 16 weeks of age. 
A
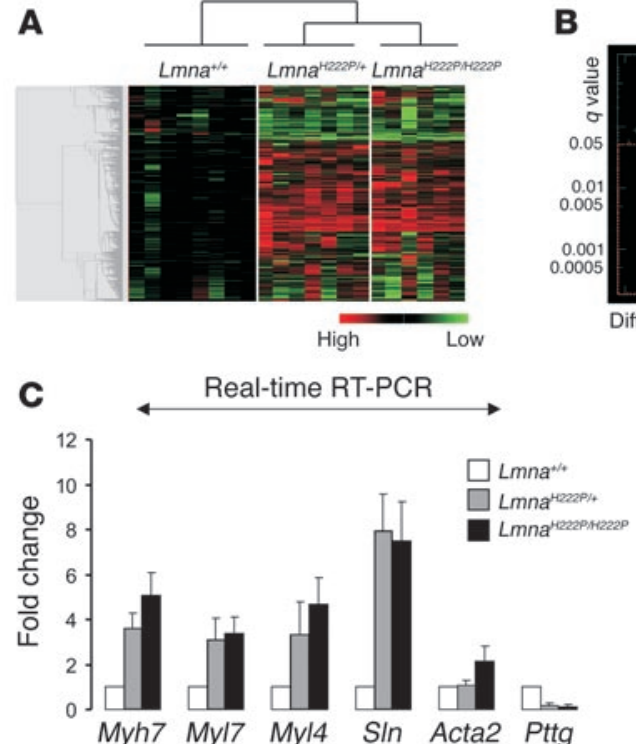
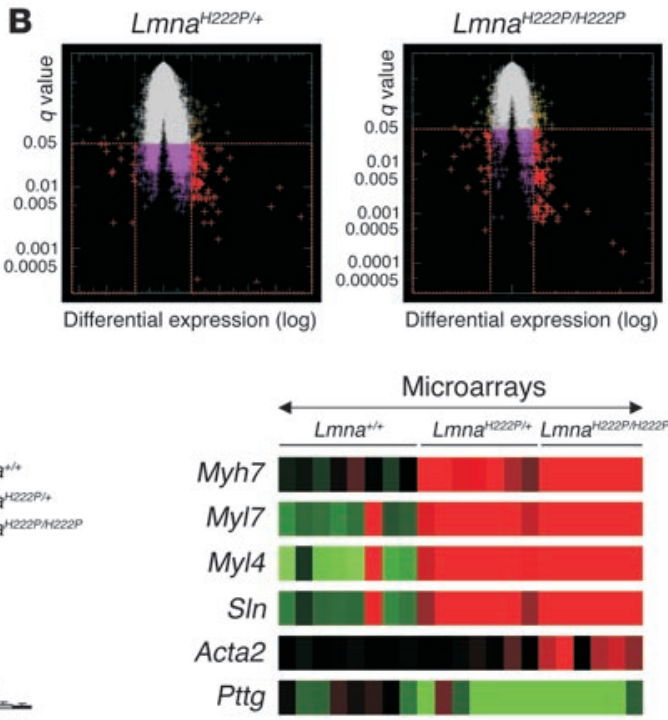

\section{Figure 1}

RNA expression profiling in hearts of Lmna H222P mice. (A) Hierarchical clustering analysis of differentially expressed genes in hearts from $\mathrm{Lmna}^{+/+}, \mathrm{Lmna} \mathrm{H}^{\mathrm{H} 222 \mathrm{P} /+}$, and $\mathrm{Lmna} \mathrm{H}^{\mathrm{H} 222 \mathrm{P} / \mathrm{H} 222 \mathrm{P}}$ mice. Rows indicate the individual genes expressed and columns indicate each sample. A color is assigned to each gene depending on its expression (red indicates higher expression and green indicates lower expression). Transcriptional profiles of hearts of $L m n a^{H 222 P / H 222 P}$ and $L m n a^{H 222 P /+}$ mice show a greater degree of similarity to each other than to hearts of control $L m n a^{+/+}$ mice. (B) Volcano plots of absolute expression values $\left(\log _{2}[q\right.$ value]) determined by robust multichip analysis. For each probe set, expression in hearts from $L m n a^{H 222 P / H 222 P}$ and $L m n a^{H 222 P /+}$ mice is plotted. A 2-fold threshold and $q<0.05$ were used to determine the probe sets significantly altered in the analysis (red). (C) Validation of RNA expression profiling of selected genes in hearts from $L m n a^{+/+}, L_{m n a}{ }^{H 222 P /+}$, and $L m n a^{H 222 P / H 222 P}$ mice using real-time PCR. Bars indicate the fold overexpression of the indicated mRNA in hearts as calculated by the $\triangle \delta \mathrm{C}_{\mathrm{T}}$ method. Values are mean \pm SD for $n=6$ samples per group. Real-time PCR was performed in triplicate with the different RNA samples. Matrices visualizing Affymetrix GeneChip data of corresponding probe sets of RNAs are shown at right of bar graph. In these matrices, each probe set is visualized as a row of colored squares with 1 square for each sample. Myh7, Myh4, Myl7, Acta2, and SIn show higher expression (red) and Pttg lower expression (green) compared with controls.

Male mice die between 4 and 9 months of age. To focus on primary events and avoid interference caused by fibrotic cells and nonspecific tissue damage in hearts from older $L m n a^{H 222 P / H 222 P}$ mice, we analyzed samples from mice at 10 weeks of age. There are no histological detectable abnormalities in hearts of mice at this age (see below). We also used hearts from heterozygous $L m n a^{H 222 P /+}$ mice, which develop signs of cardiomyopathy at 24 months of age (Bonne et al., unpublished observations) and have normal life spans.

Hearts were isolated and transcription profiles determined using amplified RNA for microarray analyses. We used Affymetrix Mouse Genome 4302.0 Arrays, which contain 45,101 probes sets for known and predicted genes. We examined similarities in transcription profiles between hearts from control $\mathrm{Lmna}^{+/+}, \mathrm{Lmna}^{\mathrm{H} 222 \mathrm{P} /+}$, and $L m n a^{H 222 P / H 222 P}$ mice by hierarchical cluster analysis. Using hearts from control mice $(n=8)$ as a baseline, this analysis revealed a strong consistency between replicates and distinct patterns of gene expression (Figure 1A). Compared with the mean value of expression in controls, hearts from $L_{m n a^{H 222 P / H 222 P}}(n=6)$ and $L m n a^{H 222 P /+}$ $(n=7)$ mice exhibited a large cluster of genes with increased expression and a small cluster with decreased expression.

We used a supervised learning method to distinguish probe sets representing genes with significant differences in expression between hearts from control and mutant mice. Probe sets were selected using sufficiently high absolute changes measured by $q$ values $(q<0.05)$, which were determined using gene-wise $t$ tests. The analysis was tuned such that the false discovery rate among probe sets identi- fied as significant was $5 \%$ and expression was more than 2 -fold different from control. This analysis yielded 104 probe sets in hearts from $L m n a^{H 222 P /+}$ mice and 114 in hearts from $L m n a^{H 222 P / H 222 P}$ mice (Figure 1B). The 104 probes sets identified in hearts from $L m n a^{H 222 P /+}$ mice corresponded to 92 upregulated genes, 69 known genes, and 23 cDNAs with unknown functions (Supplemental Table 1; supplemental material available online with this article; doi:10.1172/ JCI29042DS1). The 12 downregulated genes included 6 known genes and 6 uncharacterized cDNAs. The number of upregulated genes corresponding to the probe sets identified in hearts from $L m n a^{H 222 P / H 222 P}$ mice was 94, which included 73 known genes and 21 cDNAs of unknown function (Supplemental Table 2). The number of downregulated genes was 20 , including 8 known genes and 12 cDNAs with unknown functions. There were 57 similar probe sets between hearts from $L m n a^{H 222 P / H 222 P}$ and $L m n a^{H 222 P /+}$ mice (Supplemental Table 3).

To validate expression of selected transcripts identified by microarray analysis, we performed real-time PCR using RNA extracted from mouse hearts (Figure 1C). Genes encoding heavy and light chains of myosins (Myl7, Myl4, Myb7), actin- $\alpha 2$ (Acta2), sarcolipin $(\operatorname{Sln})$, and pituitary tumor-transforming 1 (Pttg1) were selected as representative. There was a correlation between realtime PCR results and altered expression detected by microarrays for these genes with greater than 2-fold differences in expression in hearts from both $L m n a^{H 222 P /+}$ and $L m n a^{H 222 P / H 222 P}$ mice (Figure $1 \mathrm{C}$ ).

Many genes, including some muscle-specific genes, were identical in hearts from $L m n a^{H 222 P /+}$ and $L m n a^{H 222 P / H 222 P}$ mice, with significantly 


\section{Table 1}

Top-scoring gene ontology terms in hearts of $L m n a^{H 222 P / H 222 P}$ and $L m n a^{H 222 P /+}$ mice

GO term

Inflammation

MHC class II receptor activity

Antigen processing

Antigen presentation

MHC class I receptor activity

Complement activation

Fibrosis

VEGF receptor activity

Muscle components

Contractile fiber

Sarcomere

Muscle myosin

Structural constituent of muscle

\section{Transcription/translation}

poly $(A)$ binding

Specific RNA polymerase II transcription factor activity

Single-stranded DNA binding

Eukaryotic 43S preinitiation complex

Heterogeneous nuclear ribonucleoprotein complex

ATP-dependent RNA helicase activity

Transcriptional repressor complex

tRNA ligase activity

Double-stranded RNA binding

Regulation of translational initiation

\section{Signaling pathways}

Insulin-like growth factor binding

Ras protein signal transduction

Wnt receptor signaling pathway

Heterotrimeric G-protein complex

JNK cascade

MAPK activity

Nuclear translocation of MAPK

Protein phosphatase type 2A activity

Transmembrane receptor protein kinase activity
GO identification number

H222P/H222P

0.00000015

0.00000022

0.00000036

0.00343037

0.02981152

G0:0005021

G0:0043292

G0:0030017

G0:0005859

G0:0008307

G0:0008143

G0:0003704

G0:0003697

G0:0016282

G0:0030530

G0:0004004

G0:0017053

G0:0004812

G0:0003725

G0:0006446

G0:0005520

G0:0007265

G0:0016055

G0:0005834

G0:0007254

G0:0004707

G0:0000189

G0:0000158

G0:0019199 $q$ value

$H 222 P /+$

GO, gene ontology. ERmineJ uses $q$ values to determine which gene sets are statistically significant between hearts from $L m n a^{+/+}$and $L m n a^{H 222 P / H 222 P}$ and hearts from $\mathrm{Lmna}^{+/+}$and $\mathrm{Lmna} \mathrm{H}^{\mathrm{H} 22 \mathrm{P} /+}$.

altered expression compared with those of controls (Supplemental Table 3). Myl4, Myl7, Myb7, and Sln were upregulated in hearts from both $L m n a^{H 222 P / H 222 P}$ and $L m n a^{H 222 P /+}$ mice. There was also increased expression of genes encoding LIM domain family members, including Pdlim 3 and Fbl1. However, it appeared that increased expression of muscle-specific genes was greater in hearts of $L m n a^{H 222 P / H 222 P}$ mice than in $\mathrm{Lmna}^{\mathrm{H222P/+}}$ mice (Supplemental Tables 2 and 1, respectively). Statistically significant increases in RNA transcripts encoding atrial natriuretic factor and actin- $\alpha 2$ were observed only in hearts from Lmna ${ }^{H 222 P / H 222 P}$ mice (Supplemental Table 2).

We used ermineJ software, which analyzes gene ontology terms applied to genes, to identify functional classes of genes differentially expressed in hearts of $\mathrm{Lmna}^{\mathrm{H} 222 \mathrm{P} /+}$ and $\mathrm{Lmna}^{\mathrm{H} 222 \mathrm{P} / \mathrm{H} 222 \mathrm{P}}$ mice compared with those expressed in controls. Analysis using functional class scoring improves sensitivity by statistically evaluating genes in biologically meaningful groups. In hearts from $\mathrm{Lmna}^{\mathrm{H} 222 \mathrm{P} / \mathrm{H} 222 \mathrm{P}}$ mice, the highest scoring gene ontology classes were genes encoding proteins involved in inflammation and fibrosis (Table 1). However, these classes were not significantly altered in hearts from $L m n a^{H 222 P /+}$ mice. Differential expression of genes encoding muscle components, including myosins and sarcomeric proteins, achieved statistical significance in hearts from $L m n a^{H 222 P / H 222 P}$ and $L m n a^{H 222 P /+}$ mice (Table 1). Genes encoding various proteins involved in transcription and translation also demonstrated significant differences in expression, some only in hearts of $L m n a^{H 222 P / H 222 P}$ mice and others in hearts of both $L m n a^{H 222 P / H 222 P}$ and heterozygotes (Table 1). Genes encoding proteins in the Wnt receptor signaling pathway, in heterotrimeric G-protein complexes, in the JNK cascade branch of the MAPK pathway, with protein phosphatase type $2 \mathrm{~A}$ activities, and with transmembrane receptor protein kinase activities demonstrated significantly altered expression in hearts of $L m n a^{H 222 P /+}$ and $L m n a^{H 222 P / H 222 P}$ mice (Table 1). 
A
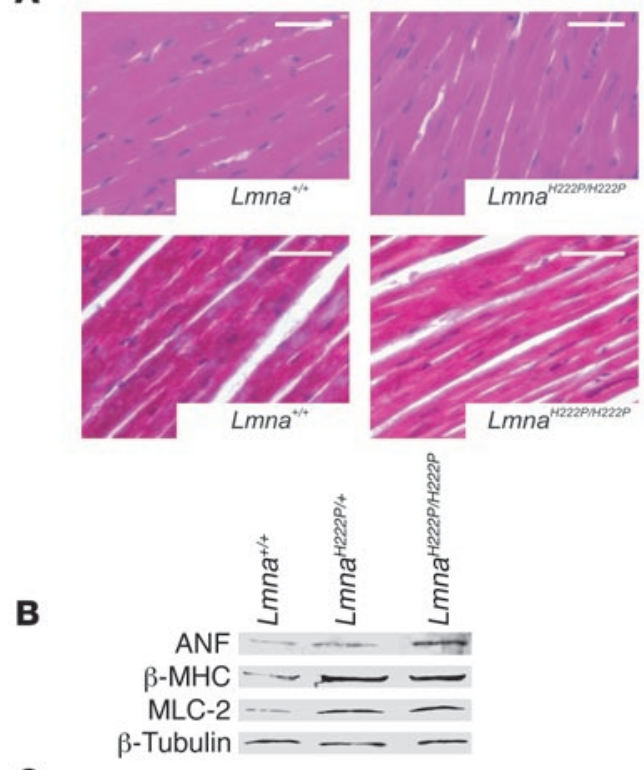

C

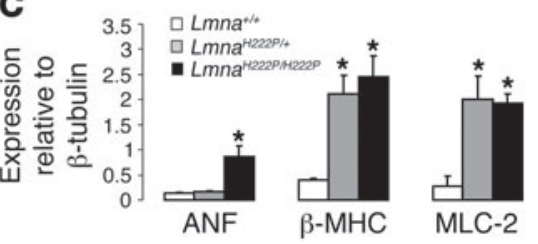

Analysis of markers of cardiomyopathy in hearts from mice with Lmna H222P mutation. Several genes discovered through microarray analysis to be differentially expressed in hearts of Lmna H222P knockin mice appeared to be involved in pathological changes of cardiomyopathy. Activation of genes encoding proteins involved in inflammation and fibrosis has been reported in previous studies of cardiomyopathies in humans and mice $(15,16)$ and is consistent with pathological changes that develop in hearts of $L m n a^{H 222 P / H 222 P}$ mice (14). However, histological examination did not reveal inflammation or fibrosis in hearts from $L m n a^{H 222 P / H 222 P}$ mice at 10 weeks (Figure 2A). This suggests that detection of molecular signatures using microarrays is more sensitive than conventional histology in detecting inflammation and fibrosis.

Deregulation of genes encoding muscle components and involved in muscle organization has been described in other studies of dilated cardiomyopathies $(17,18)$. We therefore used immunoblotting to analyze expression of $\beta$-myosin heavy chain ( $\beta$-MHC) and myosin light chain 2 (MLC-2) polypeptides, as their genes showed significantly increased expression in hearts from mutant mice. Hearts from $L m n a^{H 222 P /+}$ and $L m n a^{H 222 P / H 222 P}$ mice had 5.3-fold and 6.1-fold increases in $\beta$-MHC and 7.1-fold and 6.8-fold increases in MLC-2 expression, respectively, compared with controls (Figure 2B). We also measured expression of atrial natriuretic factor (ANF), which is upregulated in heart failure, and its expression was increased in hearts from $L m n a^{H 222 P / H 222 P}$ mice by approximately 6 -fold compared with controls (Figure 2B). However, the increase of ANF was not statistically significant in hearts from $\mathrm{Lmna}^{\mathrm{H} 222 \mathrm{P} /+}$ mice. This is consistent with results of microarray analysis and cardiac chamber dilation in hearts of $L m n a^{H 222 P / H 222 P}$ mice at 8 weeks of age (14).

MAPK pathways involved in development of cardiomyopathy are activated in hearts of Lmna H222P mice. Our functional class scoring analysis

\section{Figure 2}

Histological analysis of heart muscle in Lmna H222P mice and expression of myosins and ANF. (A) Histological analysis of hearts from 10-week-old control $L m n a^{+/+}$and $L m n a^{H 222 P / H 222 P}$ mice. Representative fixed sections of left ventricles stained with H\&E (upper panels) and Gomori's trichrome (lower panels) are shown. Note normal-appearing cardiomyocytes and absence of fibrosis. Scale bars: $50 \mu \mathrm{m}$. (B) Expression of myosins and ANF in hearts of 10-week-old $\mathrm{Lmna}^{+/+}$, $L m n a^{H 222 P /+}$, and $L m n a^{H 222 P / H 222 P}$ mice. Representative immunoblots for ANF, $\beta$-MHC, and MLC-2 are shown. $\beta$-tubulin Ab was used as a loading control. Data in bar graphs are mean \pm SD for 5 samples per group $\left({ }^{*} P<0.05\right)$.

revealed significant differences in expression of genes encoding proteins in MAPK pathways in Lmna H222P mouse hearts (Table 1). Individual genes in MAPK pathways with significantly different expressions $(q<0.05)$ in hearts from $L m n a^{H 222 P+}$ and $L m n a^{H 222 P / H 222 P}$ mice, as identified using the Database for Annotation, Visualization and Integrated Discovery (DAVID) Bioinformatics Database (http://david.abcc.ncifcrf.gov/), are listed in Supplemental Tables 4 and 5. Because enhanced JNK cascade activity, a branch of MAPK pathways, has been shown to cause cardiomyopathy and conduction defects $(19,20)$, we focused our attention on the MAPK pathways. We first evaluated phosphorylation of 2 MAPKs, JNK and ERK1/2 (pJNK and pERK1/2, respectively), in hearts from $L m n a^{+/+}$, $L m n a^{H 222 P /+}$, and $L m n a^{H 222 P / H 222 P}$ mice. These kinases are activated by phosphorylation. Immunoblotting with anti-pJNK Ab demonstrated 5-fold and 9-fold increases in pJNK in hearts from $L m n a^{H 222 P /+}$ and $L m n a^{H 222 P / H 222 P}$, respectively (Figure 3A). pERK1/2 was also significantly increased in hearts from $L m n a^{H 222 P /+}$ and $L m n a^{H 222 P / H 222 P}$ mice (2.3-fold and 2.1-fold, respectively) (Figure 3A). Infiltration of cells other than cardiomyocytes could be a potential confounding variable accounting for detection of activated MAPKs in heart tissue. To remove the influence of such cells, we tested whether JNK and ERK1/2 were activated in isolated ventricular cardiomyocytes from Lmna ${ }^{H 222 P / H 222 P}$. Expressions of pERK1/2 and $\mathrm{pJNK}$ were increased 4-fold and 12-fold, respectively, in cardiomyocytes from $L m n a^{H 222 P / H 222 P}$ mice compared to those from $\mathrm{Lmna}^{+/+}$mice (Figure 3A).

pJNK and pERK1/2 activate a series of downstream target genes, including those encoding bcl-2, Elk1 and c-Jun (21-23). Immunoblotting with Abs against bcl-2 and Elk1 demonstrated increased expression of these proteins in hearts from both $L m n a^{H 222 P / H 222 P}$ and $L m n a^{H 222 P /+}$ mice compared with $L m n a^{+/+}$mice (Figure $3 \mathrm{~B}$ ). pc-Jun was also increased in hearts from $L m n a^{H 222 P / H 222 P}$ but not $L m n a^{H 222 P /+}$ mice (Figure 3B). Increases in Elk1, bcl-2, and pc-Jun were also detected in isolated ventricular cardiomyocytes from $L m n a^{H 222 P / H 222 P}$ mice compared with controls (Figure $3 \mathrm{~B}$ ). These data indicate aberrant activation of MAPK signaling in hearts from both $\mathrm{Lmna}^{\mathrm{H} 222 \mathrm{P} / \mathrm{H} 222 \mathrm{P}}$ and $\mathrm{Lmna^{H } 2 2 2 \mathrm { P } / +}$ mice. However, the degree of enhanced signaling appeared to be greater in hearts from homozygous mutant mice.

To analyze in vivo activation of MAPK, we used an Ab that recognized pERK1/2 in sections of heart tissue. Immunofluorescence staining of heart sections from $L m n a^{+/+}$mice with these antibodies revealed a faint, rather diffuse pattern, whereas fluorescence in hearts from $L m n a^{H 222 P / H 222 P}$ mice was more intense and predominantly nuclear (Figure 4A). Quantitative analysis of individual cardiomyocytes in the sections confirmed that anti-pERK Ab labeled both cytoplasm and nucleus in hearts from $\mathrm{Lmna}^{+/+}$mice but essentially only the nucleus in hearts from $L m n a^{H 222 P / H 222 P}$ mice (Figure $4 \mathrm{~B}$ ). 
A
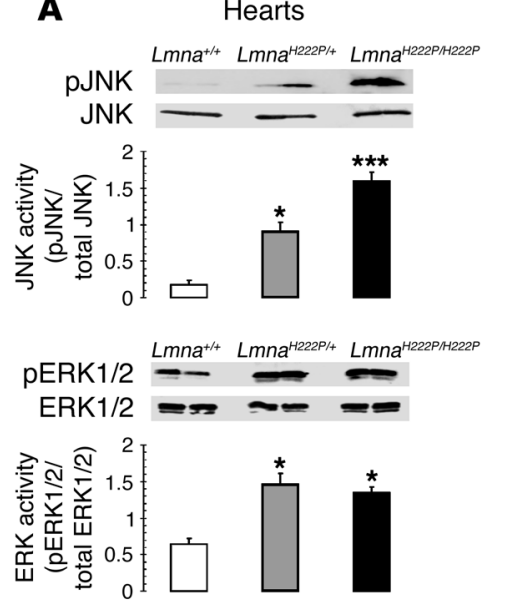

B

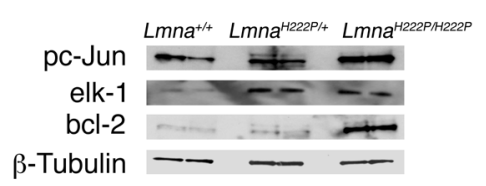

Isolated cardiomyocytes

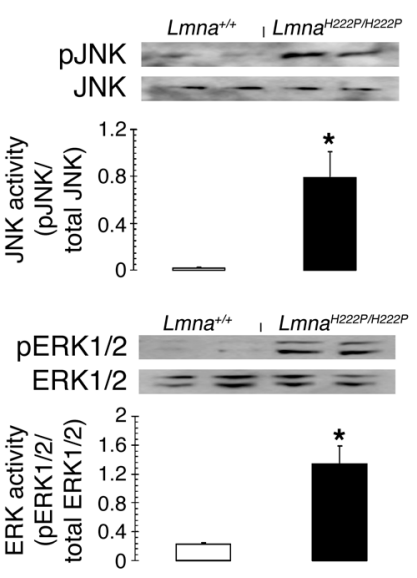

Isolated cardiomyocytes

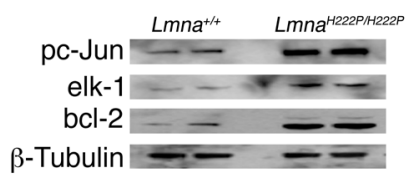

\section{Figure 3}

MAPK signaling is activated in hearts and isolated cardiomyocytes from Lmna H222P mice. (A) Detection of phosphorylated JNK (pJNK) and ERK1/2 (pERK1/2) in hearts and isolated cardiomyocytes from $\mathrm{Lmna}^{+/+}$, $\mathrm{Lmna}^{\mathrm{H} 222 \mathrm{P} /+}$, and $\mathrm{Lmna}{ }^{\mathrm{H} 222 \mathrm{P} / \mathrm{H} 222 \mathrm{P}}$ mice. JNK and ERK $1 / 2$ were measured by immunoblotting with Abs against total protein (JNK and ERK1/2) and phosphoprotein (pJNK and pERK1/2). Data in bar graphs are mean \pm SD of 5 samples per group $\left({ }^{\star} P<0.05\right.$, $\left.{ }^{* \star *} P<0.0005\right)$. (B) Effect of MAPK activation on downstream targets in $\mathrm{Lmna}^{+/+}, \mathrm{Lmna}^{\mathrm{H} 222 \mathrm{P} /+}$, and $\mathrm{Lmna} \mathrm{H}^{\mathrm{H} 22 \mathrm{P} / \mathrm{H} 222 \mathrm{P}}$ mice. Representative immunoblots using Abs that recognize phosphorylated c-Jun (pc-Jun), Elk1, and bcl-2 and $\beta$-tubulin as a loading control are shown for proteins extracted from heart tissue and isolated ventricular cardiomyocytes.
Fluorescence intensity of nuclear labeling was significantly higher in heart cells of $L m n a^{H 222 P / H 222 P}$ mice compared with $L m n a^{+/+}$mice (Figure 4C). These results demonstrate greater activation and nuclear translocation of a MAPK in hearts from $L m n a^{H 222 P / H 222 P}$ mice compared with controls.

To determine whether MAPK activation is observed only in the heart, we measured expression of downstream target genes Elk1, JunD, c-Jun, and Elk4 in different tissues. Real-time PCR showed significantly increased expression mostly in hearts from $L m n a^{H 222 P / H 222 P}$ mice (Figure 5). There was also increased expression of Elk4 in skeletal muscle of $L m n a^{H 222 P / H 222 P}$ mice.

Our initial analyses used hearts from mice 10 weeks of age, when $L m n a^{H 222 P / H 222 P}$ mice already have slight ventricular dilatation (14). Activation of fibrosis genes was also detected at this time. These alterations could affect cardiac cells and secondarily stimulate MAPK cascades. We therefore use real-time PCR to analyze expression of c-Jun, Elk1,JunD, and Elk4 in hearts from $\mathrm{Lmna}^{+/+}$and $L m n a^{H 222 P / H 222 P}$ mice at 4, 7, and 10 weeks of age. In hearts from 10 -week-old $L m n a^{H 222 P / H 222 P}$ mice, there was activation of Vegf, a marker of fibrosis, as well as Myl7 and Sln. Expression of c-Jun, Elk1, JunD, and Elk4 was also significantly increased (Figure 6). In 7-week-old $L m n a^{H 222 P / H 222 P}$ mice, there was increased cardiac expression of Myl7 but not Vegfor Sln, and expression of $c$-Jun, Elk1, $J u n D$, and Elk4 was still increased (Figure 6). At 4 weeks of age, there was only increased cardiac JunD expression in Lmna ${ }^{H 222 P / H 222 P}$ mice (Figure 6). These results show that MAPK activation precedes increased expression of a fibrosis marker and 2 muscle-specific genes in hearts of $L m n a^{H 222 P / H 222 P}$ mice.

Expression of Lamin A with the $H 222 P$ aa substitution activates JNK and ERK and alters their subcellular localization. To determine whether expression of Lamin A with the H222P aa substitution is responsible for activation of MAPK signaling, we measured pERK1/2 and pJNK in transiently transfected Cos-7 and C2C12 cells expressing GFP fusions of wild-type and H222P Lamin A. Immunoblotting with Abs against total ERK1/2 and pERK1/2 demonstrated that expression of H222P Lamin A increased the amount of phosphorylated protein (Figure 7, A and B). The increase was significant compared with that of nontransfected cells and cells expressing the GFP fusion of wild-type Lamin A.

Translocation of pERK1/2 and pJNK from cytoplasm to nucleus is required for activation of downstream targets $(24,25)$. In nontransfected Cos-7 and C2C12 cells, pERK1/2 was mainly distributed in the cytoplasm (Figure 7, C and D). When transfected with a plasmid expressing the GFP fusion of wild-type Lamin A, pERK1/2 was also mainly distributed in cytoplasm (Figure 7, C and D). In contrast, expression of a GFP fusion of H222P Lamin A induced translocation of pERK1/2 into the nucleus (Figure 7, C and D). Approximately $80 \%$ of Cos-7 and C2C12 cells expressing H222P Lamin A showed a nuclear localization of pERK1/2. Nuclear localization of pERK $1 / 2$ was observed in only $15 \%$ of Cos- 7 cells and $30 \%$ of $\mathrm{C} 2 \mathrm{C} 12$ cells expressing wild-type Lamin A and was not observed in untransfected cells (Figure 7, E and F). Similar results were obtained for pJNK (Supplemental Figure 1). Hence, expression of H222P Lamin A stimulates phosphorylation and nuclear translocation of JNK and ERK1/2. While MAPKs were activated in C2C12 myoblasts transfected with H222P Lamin A, activated MAPK was not detected in skeletal muscle from $L m n a^{H 222 P / H 222 P}$ mice. However, myoblasts are only a small component of heterogeneous skeletal muscle sections. Furthermore, in humans with EDMD and in $L m n a^{H 222 P / H 222 P}$ mice, skeletal muscle is variably affected.

Expression of Lamin A with a substitutions identified in EDMD associated with cardiomyopathy activates JNK and ERK. To further evaluate the effects of Lamin A mutants on activation of MAPK pathways, we examined expression of c-Jun and Elk1 reporter genes. We transiently transfected Cos-7 cells with plasmids encoding GFP fusions of H222P Lamin A as well as wild-type Lamin A and 5 other Lamin A mutants. Cells were simultaneously transfected with plasmids encoding a reporter system to detect c-Jun or Elk1 promoter activities. Expression of H222P Lamin A and Lamin A with the N195K, R298C, and N456I aa substitutions found in EDMD significantly increased activity of the c-Jun and Elk1 promoters (Figure 8). Overexpression of wild-type Lamin A and 
A

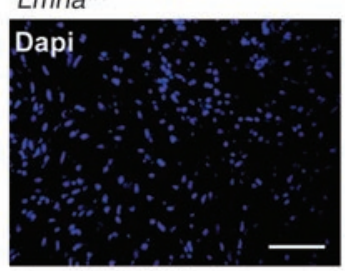

Lmna

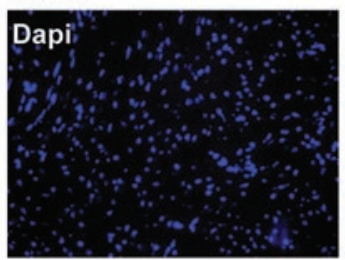

B

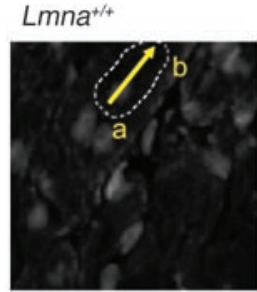

Lmna ${ }^{\text {H222P/H222P }}$

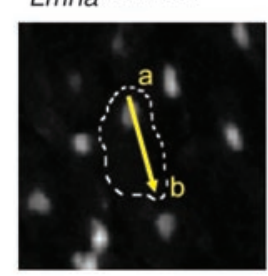

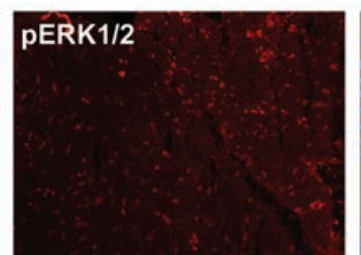

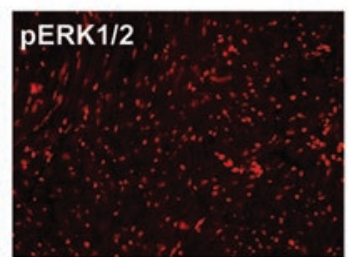

C
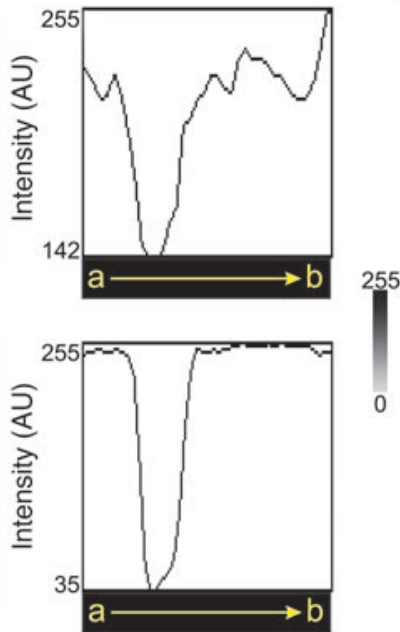
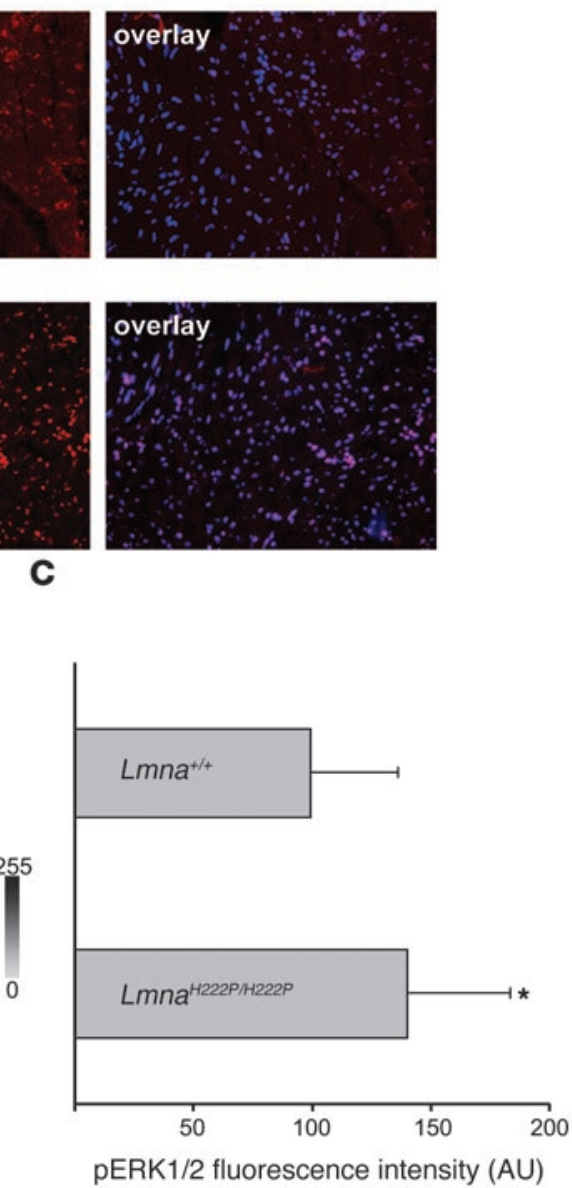

\section{Figure 4}

Immunofluorescence microscopic analysis of $\mathrm{pERK} 1 / 2$ in heart sections from LmnaH222P/H222P mice. (A) Sections of frozen heart from $L m n a^{+/+}$ (top panel) and $L m n a^{H 222 P / H 222 P}$ (bottom panel) mice were analyzed by immunofluorescence microscopy using $A b$ recognizing $p E R K 1 / 2$. Sections were counterstained with DAPI. Scale bars: $50 \mu \mathrm{m}$. (B) Quantification of $p E R K 1 / 2$ labeling in cardiomyocytes from $\mathrm{Lmna}^{+/+}$mice and LmnaH222P/H222P mice. Cardiomyocytes are delimited by dotted line and intensity of emitted fluorescence is measured along the yellow line ( $a$ to $b$ ). Position of the nucleus and intensity of fluorescence using anti $p E R K 1 / 2 A b$ is shown in the diagram of a single cardiomyocyte. (C) Bars indicate intensity of $\mathrm{pERK} 1 / 2$ fluorescence in the nucleus of $L m n a^{+/+}$ and $L m n a^{H 222 P / H 222 P}$ cardiomyocytes. Values are mean \pm SD from 90 cardiomyocytes from 2 different hearts per group $\left({ }^{\star} P<0.05\right)$.
Lamin A with a R482W mutation found in subjects with Dunnigan-type partial lipodystrophy did not significantly increase their activity. However, expression of 1 Lamin A with an aa substitution that causes EDMD (T528K) did not significantly increase $c$-Jun and Elk1 promoters activities in this assay. A possible explanation of this observation may be that a GFP fusion of this mutant folds abnormally or is not as stable as the others when overexpressed in transfected cells. These results show that expression of A-type lamins with aa substitutions encoded by LMNA mutations causing cardiomyopathy leads to stimulation of downstream target genes in MAPK cascades in cultured cells.

\section{Discussion}

Although the causative genetic mutations have been identified and the descriptive pathology well documented, cellular mechanisms linking mutations in $L M N A$ to cardiomyopathy are unknown. While several investigators have hypothesized that LMNA mutations lead to alterations in gene expression that could have tissue-selective pathogenic consequences (7), altered expression of functional groups of genes or activation of signal transduction pathways that might explain the development of disease have not been demonstrated in affected tissues. We addressed this issue by using genome-wide profiling in hearts from a mouse model of autosomal dominant EDMD. Our analysis led us to focus on MAPK signaling because in a genome-wide expression analysis, several genes related to this pathway had significantly altered expression in hearts of mice with the Lmna H222P mutation prior to development of significant cardiomyopathy.

In hearts of $L m n a^{H 222 P / H 222 P}$ mice, we found significantly increased expression of transcripts encoding several downstream components of MAPK cascades, such as c-Jun and Elk1, only by using realtime PCR. Increased expression of these transcripts, which was approximately 2 -fold, was not detected in our microarray analysis. Similar discrepancies between microarrays and real-time PCR have been reported $(26,27)$, especially when the absolute expression levels are low or when the differences between experimental and control are relatively small, which was the case for the transcripts we measured. In addition to increased expression of transcripts encoding several components, activation of MAPK cascades in hearts of Lmna $\mathrm{H} 222 \mathrm{P}$ mice was also strongly supported by increased levels of selected encoded proteins, increases in nuclear pERK1/2, and activation of ERK $1 / 2$ and JNK in cells transfected with constructs encoding Lamin A with aa substitutions that cause EDMD.

MAPKs are successively acting phosphorylases that function as regulators of cell growth, differentiation, and transformation and have been implicated in many physiological and pathological processes $(22,28,29)$. Several downstream target genes are activated by MAPKs, and we found this to be true for Elk1, Bcl-2, JunD, Elk4, and c-Jun in hearts from Lmna H222P mice. Activation of these targets can in turn regulate expression of additional genes, 


\section{C-Jun}

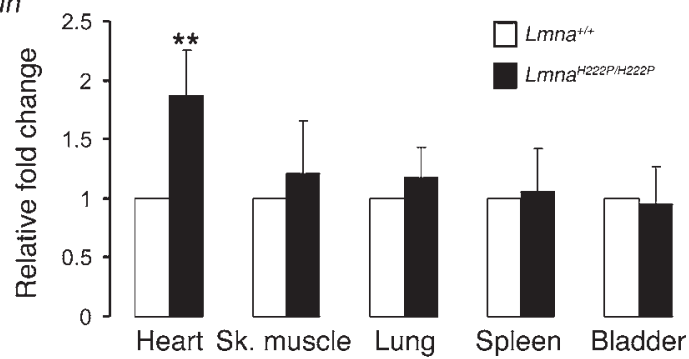

Elk1

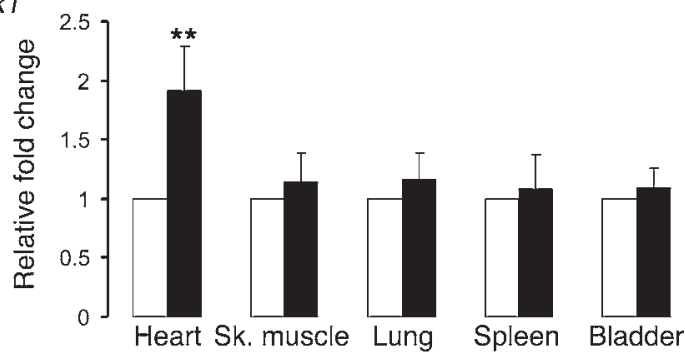

$\operatorname{JunD}$

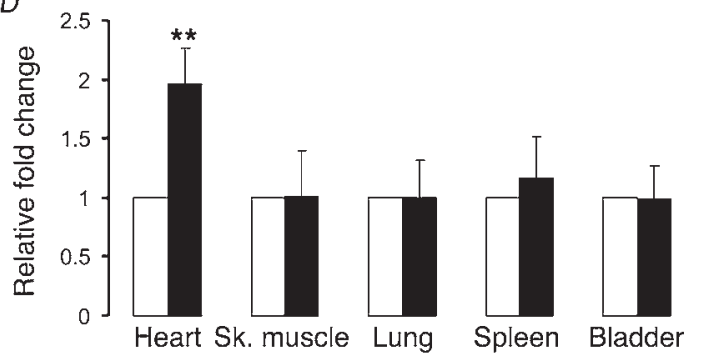

Elk4

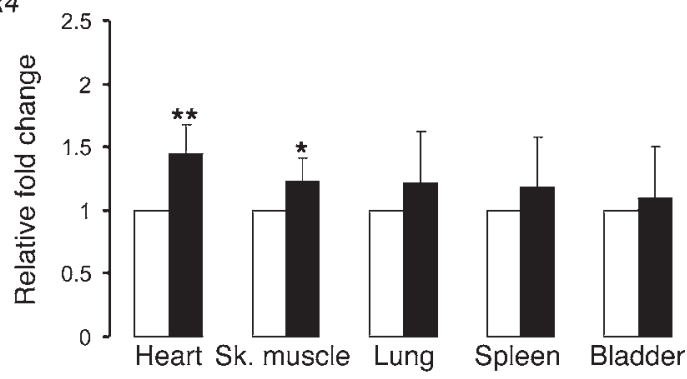

including those encoding proteins involved in sarcomere structure, cardiomyofiber organization, and other aspects of heart function $(30,31)$. Abnormal expression of these proteins can lead to cardiomyopathy (Figure 9).

MAPK activation occurred prior to significant cardiomyopathy in $L m n a^{H 222 P / H 222 P}$ mice and also in $L m n a^{H 222 P /+}$ mice, which do not develop clinical heart disease until 2 years of age. This is consistent with the hypothesis that activation of MAPK signaling underlies development of disease instead of occurring as a consequence of disease. The temporal differences in the development of cardiomyopathy in heterozygous and homozygous mice may be due to dosage, as there appeared to be more significant activation of JNK and increased expression of its downstream targets bcl-2 and phosphorylated c-Jun in hearts from $L m n a^{H 222 P / H 222 P}$ mice compared with hearts from $L m n a^{H 222 P /+}$ mice. Several genes were also activated or repressed in heterozygous mice compared with homozygous mice; however, how this is related to development of disease remains to be investigated.

\section{Figure 5}

Expression of c-Jun, Elk1, JunD, and Elk4 in various tissues from 10-week-old $\mathrm{Lmna}^{+/+}$and $\mathrm{Lmna} \mathrm{H}^{\mathrm{H} 22 \mathrm{P} / \mathrm{H} 222 \mathrm{P}}$ mice. Summary of real-time PCR results in heart, skeletal muscle (sk. muscle), lung, spleen, and bladder are shown. Bars indicate the fold overexpression of the indicated mRNA normalized to Gapdh as calculated by the $\Delta \delta \mathrm{C}_{\mathrm{T}}$ method. Values are mean \pm SD for 6 samples per group $\left({ }^{\star} P<0.05,{ }^{* *} P<0.005\right)$.

Results from previous studies have implicated activation of MAPKs in development of cardiomyopathy. Petrich et al. $(19,20)$ generated transgenic mice expressing an activated mutant of MKK7, a kinase-activating JNK, specifically in heart. These mice developed dilated cardiomyopathy. Similar results have been observed in transgenic mice overexpressing mutants of MKK3 and MKK6, kinases that also activate MAPKs (32). Nicol et al. (33) generated transgenic mice overexpressing MEK5, which activates ERK, in hearts, and these mice developed dilated cardiomyopathy. JNK is also activated in dilated human hearts $(34,35)$. Recently, Rodriguez-Viciana et al. demonstrated that mutations in MEK1 and $M E K 2$, which encode kinases that activate ERK1 and ERK2, cause cardio-facial-cutaneous syndrome (36). The MEK mutants were more active than wild type in phosphorylating ERK. Transgenic mice expressing activated MEK1 similarly have enhanced ERK1/2 signaling and develop cardiomyopathy (37). Activation of the ERK cascade has also been reported in caveolin-3 (38), caveolin-1 (39), and p85 subunit of class IA PI3K (40) knockout mice, all of which develop cardiomyopathy at 2 months of age.

While it remains unclear how A-type lamins with a substitutions activate MAPKs, our results show that they do so when expressed in transfected cells. Activation of the ERK subfamily of MAPKs is mediated by receptor protein tyrosine kinases or G-protein-coupled receptors (41). JNK subfamily of MAPK is activated by osmotic stress (42) and physical stress (43). It is possible that abnormalities in the nuclear lamina lead to activation of G-protein-coupled or other receptors via an unknown mechanism (Figure 9). Several investigators have hypothesized that alterations in response to stress may underlie the development of striated muscle diseases caused by LMNA mutations (7). Abnormal responses to stress in cells with abnormalities in A-type lamins could therefore potentially have an impact on activation of JNK (Figure 9). Fibroblasts from mice lacking A-type lamins have increased nuclear deformation and impaired viability under mechanical strain as well as attenuated NF- $\kappa \mathrm{B}$-regulated transcription in response to stress (14). In addition, we observed that expression of H222P Lamin A in transfected cells lead to enhanced nuclear translocation of activated ERK and JNK. Smith et al. (44) have demonstrated that suppression of cell proliferation after retinoic acidinduced endoderm differentiation of embryonic stem and carcinoma cells is achieved by restricting nuclear entry of activated MAPK, and an intact cytoskeleton is required for the restraint. Hence, interactions between the nuclear lamina and cytoskeletal components could potentially influence nuclear translocation of activated MAPKs, with abnormalities in the lamina enhancing their nuclear localization. Recently, Ivorra et al. (45) highlighted a direct interaction between A-type lamins and the transcription factor c-Fos. This raises the possibility that A-type lamins may bind to components of MAPK cascades and that the H222P aa substitution may alter such an interaction. This hypothesis remains to be tested.

A fetal-like gene expression program encoding cytoskeletal proteins is characteristic of many types of cardiomyopathy $(17,18,46-49)$ and is similarly initiated during cardiac remodel- 
10 weeks

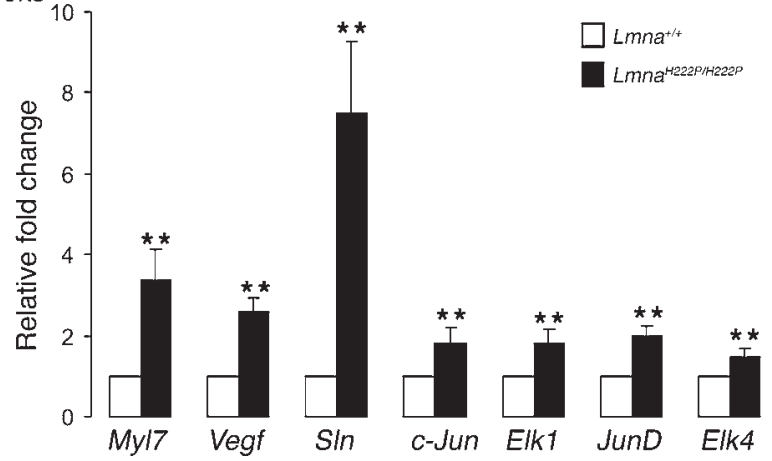

7 weeks

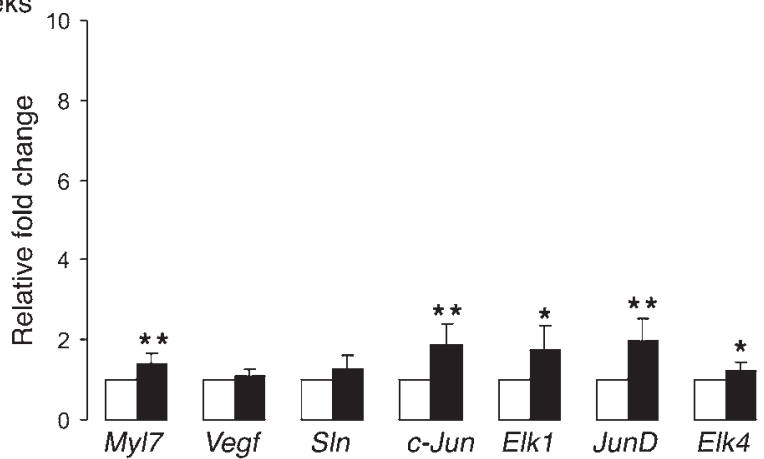

4 weeks

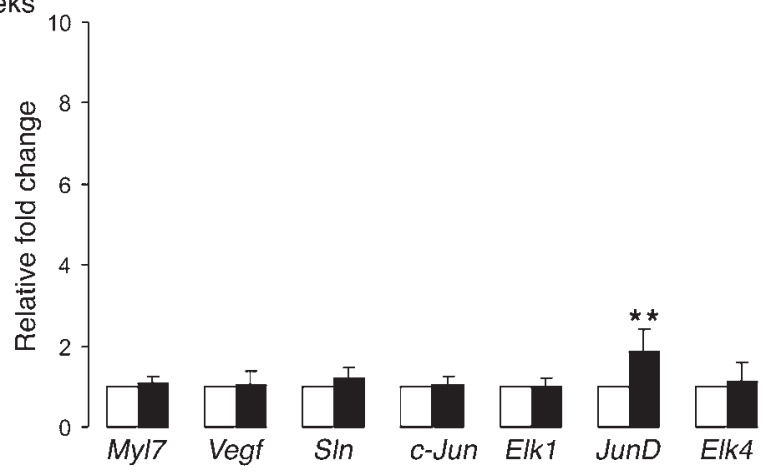

ing due to mechanical strain, such as in hypertensive cardiomyopathy $(50,51)$. Increases in ventricular expression of ANF have been documented in experimental models of heart failure and cardiomyopathy $(29,38,52-55)$ as well as in human heart failure (56). Our analysis identified 104 and 114 genes that were differentially expressed in hearts from $L m n a^{H 222 P /+}$ and $L m n a^{H 222 P / H 222 P}$ mice, respectively. These included several in the cardiac fetal gene expression program, such as those encoding $\beta$-MHC (Myb7), MLC-2 (Myl4, Myl7), and ANF (Anf). However, changes in the levels of these genes were different in hearts of $\mathrm{Lmna}^{\mathrm{H} 222 \mathrm{P} / \mathrm{+}}$ and $L m n a^{H 222 P / H 222 P}$ mice. For example, the $\log _{2}$-fold changes in expression of $M y b 7$ were 2.38 in hearts of $L m n a^{H 222 P /+}$ mice and 3.49 in hearts of $L m n a^{H 222 P / H 222 P}$. $\log _{2}$-fold changes in expression of Myl4 were 3.84 in hearts of $L m n a^{H 222 P /+}$ and 4.66 in hearts of Lmna $a^{H 222 P / H 222 P}$ mice. Anf was not differentially expressed in hearts of $\mathrm{Lmna}^{\mathrm{H} 222 \mathrm{P} /{ }_{+}}$mice compared with wild-type mice but was upregulated significantly in hearts of $L m n a^{H 222 P / H 222 P}$ mice. These different degrees of gene expression changes may reflect the early onset of heart failure in $L m n a^{H 222 P / H 222 P}$ mice, which develop symptoms at approximately 2 months of age. In

\section{Figure 6}

Time-course expression of genes activated by MAPK in hearts from LmnaH222P/H222P mice at 4, 7, and 10 weeks of age. Expression of Myl7, Vegf, SIn, C-Jun, Elk1, JunD, and Elk4 in hearts of Lmna ${ }^{+/+}$and $L m n a^{H 222 P / H 222 P}$ mice is shown. Bars indicate the fold overexpression of the indicated mRNA normalized to Gapdh as calculated by the $\Delta \delta \mathrm{C}_{\mathrm{T}}$ method. Values are mean \pm SD for 6 samples per group $\left({ }^{\star} P<0.05\right.$, $\left.{ }^{\star \star} P<0.005\right)$.

contrast, $L m n a^{H 222 P /+}$ mice exhibit a decrease in left ventricular fractional shortening only at 2 years of age (Bonne et al., unpublished observations).

Expression of genes encoding skeletal muscle $\alpha$-actin (57) and c-myc (58), which are reexpressed during cardiac remodeling, was not increased in hearts of $L m n a^{H 222 P /+}$ or $L m n a^{H 222 P / H 222 P}$ mice. These genes are activated in an early response against passive tension, for example in cardiac hypertrophy secondary to pressure overload. Failure of activation of these genes is consistent with our observation of dilated cardiomyopathy without cellular hypertrophy or disarray in hearts of $L m n a^{H 222 P / H 222 P}$ mice (14). The observed upregulation of genes encoding extracellular matrix proteins, such as those encoding collagen I $\alpha 2$ (Col1a2), decorin (Dcn), and matrix metalloproteinase 14 (Mmp14), may underlie the development of fibrosis in hearts of $L m n a^{H 222 P / H 222 P}$ mice.

Our results provide a foundation upon which pharmacological interventions for treatment or prevention of cardiomyopathy in EDMD can be based. If mutant A-type lamins activate JNK and ERK, which in turn lead to gene expression alterations responsible for the development of cardiomyopathy, MAPK inhibitors could potentially be used to treat or prevent disease. MAPK inhibitors have been studied as potential therapeutic agents for a wide range of diseases. JNK inhibitors have been shown to be beneficial in reducing myocardial ischemic injury (59), stroke (60), hearing impairment (61), and various neurodegenerative disorders (62). The availability of MAPK inhibitors with in vivo activities makes possible clinical trials to prevent or treat cardiomyopathy in $L m n a^{H 222 P / H 222 P}$ mice. In addition, knockout mouse models of ERK1/2 and JNK have been generated (63). Crossing those mice with $L m n a^{H 222 P / H 222 P}$ mice may also establish whether abolishing the function of MAPKs can rescue cardiomyopathy.

Analysis of genome-wide expression changes in hearts from Lmna $\mathrm{H} 222 \mathrm{P}$ mice revealed significant alterations in expression of genes involved in inflammation and fibrosis prior to detectable abnormalities in hearts examined using conventional histological methods. Ultimately, fibrosis with minimal inflammation occurs in hearts of $L m n a^{H 222 P / H 222 P}$ mice (14) as well as in hearts of Lmna ${ }^{N 195 K / N 195 K}$ mice, another model of EDMD (64). This suggests that in addition to treatment with MAPK inhibitors, early treatment with antiinflammatory or antifibrotic agents may benefit human subjects with EDMD.

\section{Methods}

Mice. Lmna H222P knockin mice were generated and genotyped as described (14). Hearts were isolated from male $L m n a^{H 222 P / H 222 P}, L m n a^{H 222 P /+}$ and $L m n a^{+/+}$mice at 4, 7, or 10 weeks of age. For all immunoblotting and real-time PCR experiments, $L m n a^{H 222 P / H 222 P}$ and $L m n a^{H 222 P /+}$ mice were compared directly with $L m n a^{+/+}$littermates. For microarray analysis, mice were taken from 5 different litters of crosses between $L m n a^{H 222 P /+}$ mice. Control $\mathrm{Lmna}^{+/+}$mice were taken from each of the litters from which $L m n a^{H 222 P / H 222 P}$ and $L m n a^{H 222 P /+}$ were used. 


\section{Figure 7}

Expression of $\mathrm{H} 222 \mathrm{P}$ Lamin $\mathrm{A}$ in transfected Cos-7 and $\mathrm{C} 2 \mathrm{C} 12$ cells leads to increased phosphorylation and enhanced nuclear translocation of ERK1/2. (A and $\mathbf{B}$ ) Effect of $\mathrm{H} 222 \mathrm{P}$ Lamin $A$ expression on levels of pERK1/2 in transfected Cos-7 (A) and C2C12 (B) cells. Immunoblotting with pERK1/2 Ab and total ERK1/2 Ab was performed. Data are shown as mean \pm SD of $11($ A) and 7 (B) samples per group $\left({ }^{*} P<0.05\right)$. Significance of the results was determined using paired Student's $t$ test (for parametric data) and a Wilcoxon rank-sum test (for nonparametric data). Immunoblotting with GFP $A b$ is shown to demonstrate expression of proteins encoded by transfected plasmids. Immunoblotting with $\beta$-actin $\mathrm{Ab}$ was used as a loading control. ( $\mathbf{C}$ and D) Effect of H222P Lamin A on nuclear translocation of $p E R K 1 / 2$ in transfected Cos-7 (C) and C2C12 (D) cells. Representative photomicrographs are shown for nontransfected (NT) cells, transfected cells expressing a GFP fusion of wild-type Lamin $A$, and transfected cells expressing a GFP fusion of Lamin A with the H222P amino acid substitution. Arrowheads show enhanced nuclear localization of $p E R K 1 / 2$ in cells expressing GFP H222P Lamin A. Scale bars: $10 \mu \mathrm{m}$. (E and F) Percentage of Cos-7 $(E)$ and $\mathrm{C} 2 \mathrm{C} 12(\mathrm{~F})$ cells with $\mathrm{pERK} 1 / 2$ primarily in the nucleus. Nontransfected cells, transfected cells expressing a GFP fusion of wild-type Lamin A, and transfected cells expressing a GFP fusion of Lamin A with the H222P aa substitution were randomly counted and scored for nuclear pERK1/2 (see arrowheads in C for example). Transfected cells were determined by presence of GFP signal. Values are mean \pm SD for 200 cells per group $\left({ }^{*} P<0.05\right)$. The person counting the cells was blinded as to which protein was expressed.

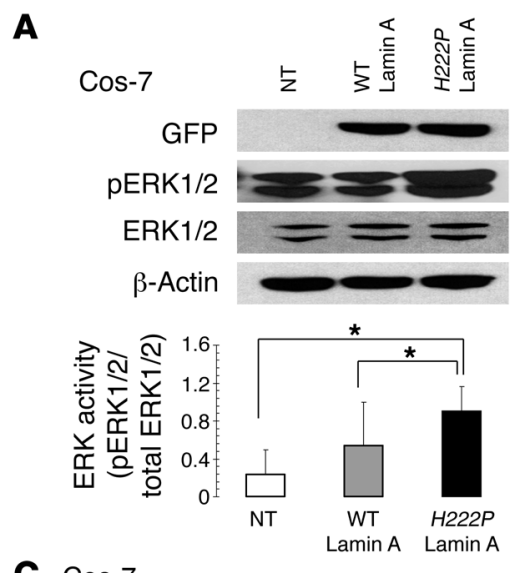

C $\operatorname{Cos}-7$

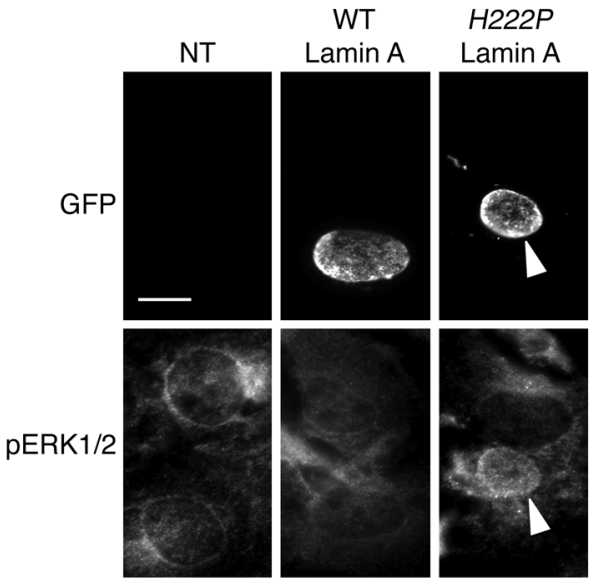

E $\operatorname{Cos}-7$

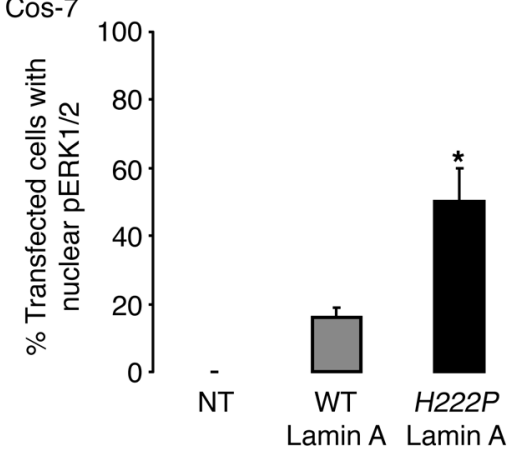

B

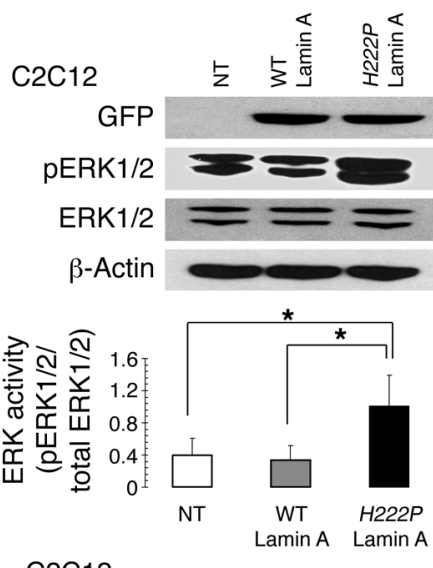

D

$\mathrm{C} 2 \mathrm{C} 12$

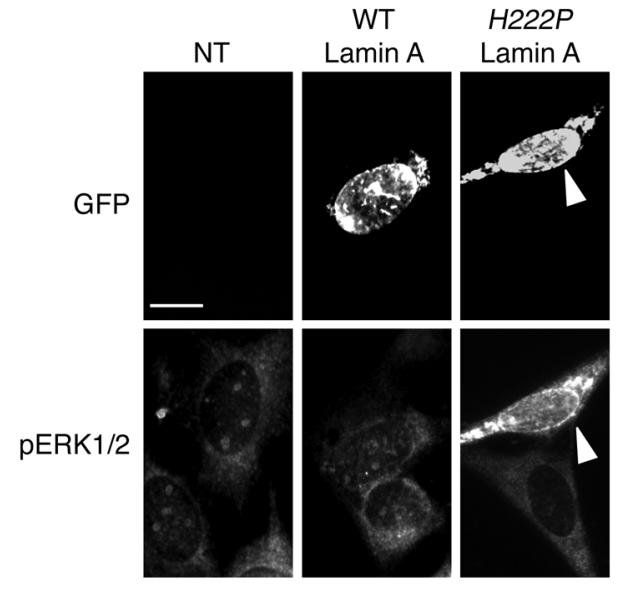

F $\quad \mathrm{C} 2 \mathrm{C} 12$

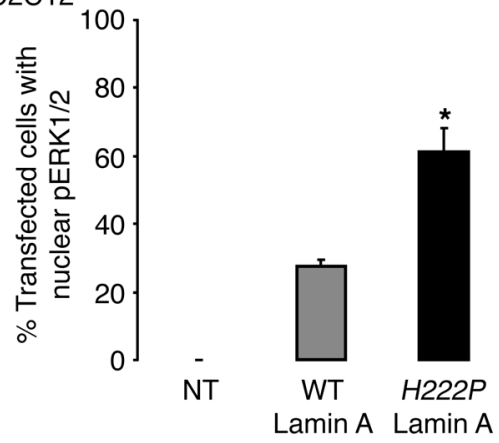

RNA isolation. Total RNA was extracted using the RNeasy isolation kit (QIAGEN) according to the manufacturer's instructions. Adequacy and integrity of extracted RNA were determined by gel electrophoresis, and concentrations were measured by ultraviolet absorbtion spectroscopy.

Microarray processing. We used GeneChip Mouse Genome 4302.0 Arrays (Affymetrix), which contain 45,101 probe sets corresponding to known genes and expressed sequence tags. Complementary DNA synthesis, cRNA synthesis, and labeling were performed as described in the Affymetrix GeneChip Expression Analysis Technical Manual. Hybridization, washing, staining, and scanning of arrays were performed at the GeneChip Core Facility of the Columbia University Genome Center.

Microarray data analysis. Image files were obtained through Affymetrix GeneChip software and analyzed by robust multichip analysis using Affymetrix microarray ".cel” image files and GeneTraffic 3.0 software (Stratagene). Robust multichip analysis is composed of 3 steps: background correction, quantile normalization, and robust probe set summary. Genes were identified as being differentially expressed if they met a false discovery rate threshold of $q<0.05$ in a 2-tailed Student's $t$ test and showed at least a 2-fold difference in expression independent of absolute signal intensity. We have made the gene expression data available in the National Center for Biotechnology Information's Gene Expression Omnibus (GEO; http://www.ncbi.nlm.nih.gov/geo/), accessible through GEO Series accession numbers GSE6397 and GSE6398.

Analysis of functional groups of genes. Gene expression changes related to functional groups were analyzed using the class score method in ermineJ (version 2.1.12; http://www.bioinformatics.ubc.ca/ermineJ/) to provide a statistical confidence to functional groupings (65). The algorithm takes as input the log-transformed 2-tailed Student's $t$ test $P$ values of genes that are members of a single gene ontology class and estimates the probability that the set of $q$ values would occur by chance. Significant gene ontology terms were identified using a false discovery rate of 


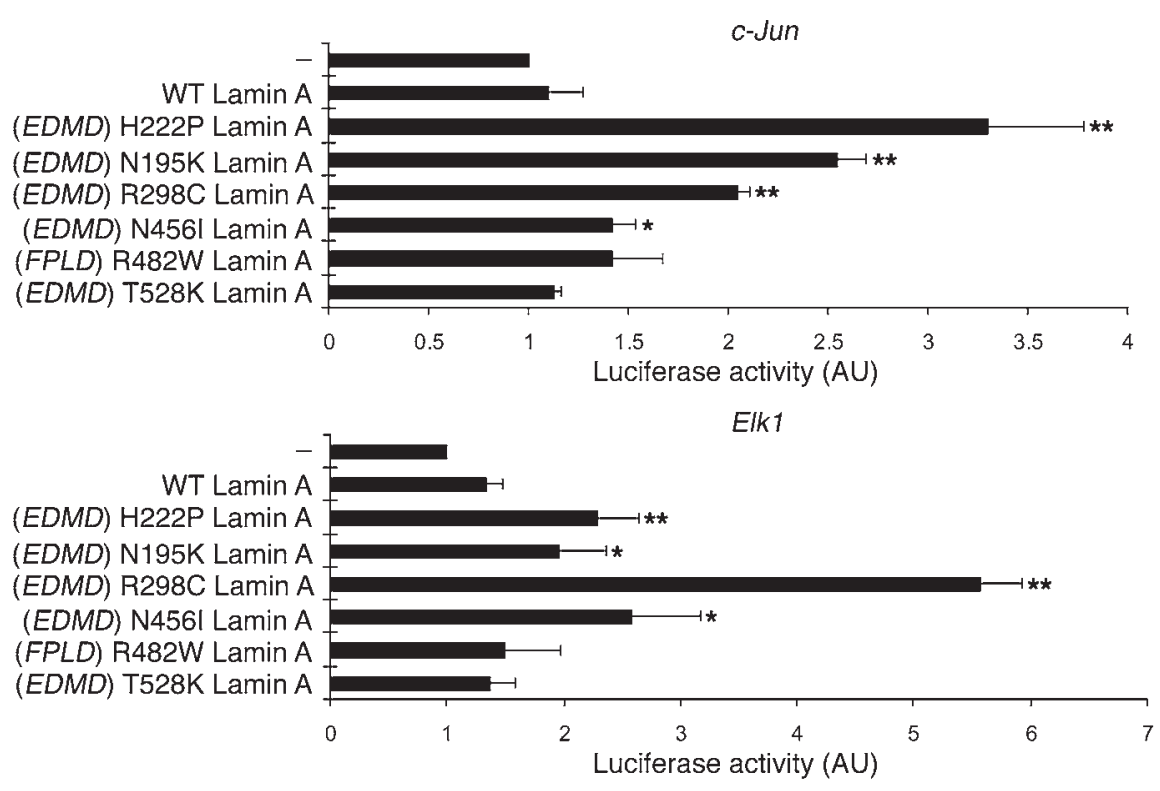

Figure 8

Activation of $c$-Jun and Elk1 by expression of Lamin A mutants. Cos-7 cells were transiently transfected with plasmids encoding wild-type Lamin A, Lamin A with the indicated amino acid substitution and the associated phenotype to each mutation (e.g., EDMD or familial partial lipodystrophy [FPLD]), or empty vector control. After 24 hours, luciferase activities induced by expression of $c$-Jun and Elk1 were measured in cell lysates and normalized to $\beta$-gal activities obtained from a protein encoded by a cotransfected plasmid. Results are mean \pm SD of 5 experiments $\left({ }^{*} P<0.05,{ }^{* *} P<0.005\right)$.
0.05. For automated functional annotation and classification of genes of interest based on gene ontology terms, we used the DAVID database (http://david.abcc.ncifcrf.gov/) (66).

Real-time PCR analysis. We synthesized cDNA using Omniscript Reverse Transcriptase (QIAGEN) on total cellular RNA. For each replicate in each experiment, RNA from tissue samples of different animals was used. Primers were designed correspond to mouse RNA sequences using Primer3 (http://frodo.wi.mit.edu/cgi-bin/primer3/primer3_www.cgi). The real-time PCR reaction contained iQ SYBR Green Supermix (Bio-Rad), $200 \mathrm{nM}$ of each primer, and $0.2 \mu \mathrm{l}$ of template in a $25-\mu \mathrm{l}$ reaction volume. Amplification was carried out using the MyiQ Single-Color Real-Time PCR Detection System (Bio-Rad) with an initial denaturation at $95^{\circ} \mathrm{C}$ for 2 minutes followed by 50 cycles at $95^{\circ} \mathrm{C}$ for 30 seconds and $62^{\circ} \mathrm{C}$ for 30 seconds. Relative levels of mRNA expression were calculated according to the $\Delta \delta C_{T}$ method (67). Individual expression values were normalized by comparison with Gapdh mRNA.

Extraction of proteins from hearts and immunoblotting. Hearts were excised from mice and snap-frozen in liquid nitrogen-cooled isopentane. To obtain protein extracts, both ventricles were homogenized in extraction buffer (25 mM Tris [pH 7.4], $150 \mathrm{mM} \mathrm{NaCl}, 5 \mathrm{mM}$ EDTA, $10 \mathrm{mM}$ sodium pyrophosphate, $1 \mathrm{mM} \mathrm{Na}_{3} \mathrm{VO}_{4}, 1 \% \mathrm{SDS}, 1 \mathrm{mM}$ dithiothreitol) containing protease inhibitors $(25 \mathrm{mg} / \mathrm{ml}$ aprotinin and $10 \mathrm{mg} / \mathrm{ml}$ leupeptin). Protein samples were subjected to SDS-PAGE, transferred to nitrocellulose membranes, and blotted with primary Abs against Elk1 (Santa Cruz Biotechnology Inc.),

\section{Figure 9}

Model of how abnormalities of A-type lamins in the nuclear lamina may lead to cardiomyopathy. Abnormalities of A-type lamins in the nuclear lamina activate MAPK cascades, possibly via heterotrimeric G-protein receptors or by inducing stress responses by unknown mechanisms (?). This leads to activation of G-proteins (RAS and RAC), protein kinase (RAF), and subsequent enhanced phosphorylation of ERK and JNK $1 / 2$, resulting in nuclear translocation. In the nucleus, pERK1/2 and pJNK activate transcription factors such as Elk1, bcl-2, JunD, c-Jun, and Elk4, leading to increased synthesis of these proteins. Increased amounts and activities of transcription factors activated by pJNK and pERK $1 / 2$ alter expression of other genes, some encoding components of muscle fibers and sarcomeres. Aberrant expression of these proteins leads to development of cardiomyopathy.
ERK1/2 (Santa Cruz Biotechnology Inc.), pERK1/2 (Cell Signaling Technology), JNK1 (Santa Cruz Biotechnology Inc.), pJNK (Cell Signaling Technology), bcl-2 (Santa Cruz Biotechnology Inc.), pc-Jun (Santa Cruz Biotechnology Inc.), $\beta-\mathrm{MHC}$ (Santa Cruz Biotechnology Inc.), MLC-2 (Santa Cruz Biotechnology Inc.), ANF (Santa Cruz Biotechnology Inc.), $\beta$-actin (Santa

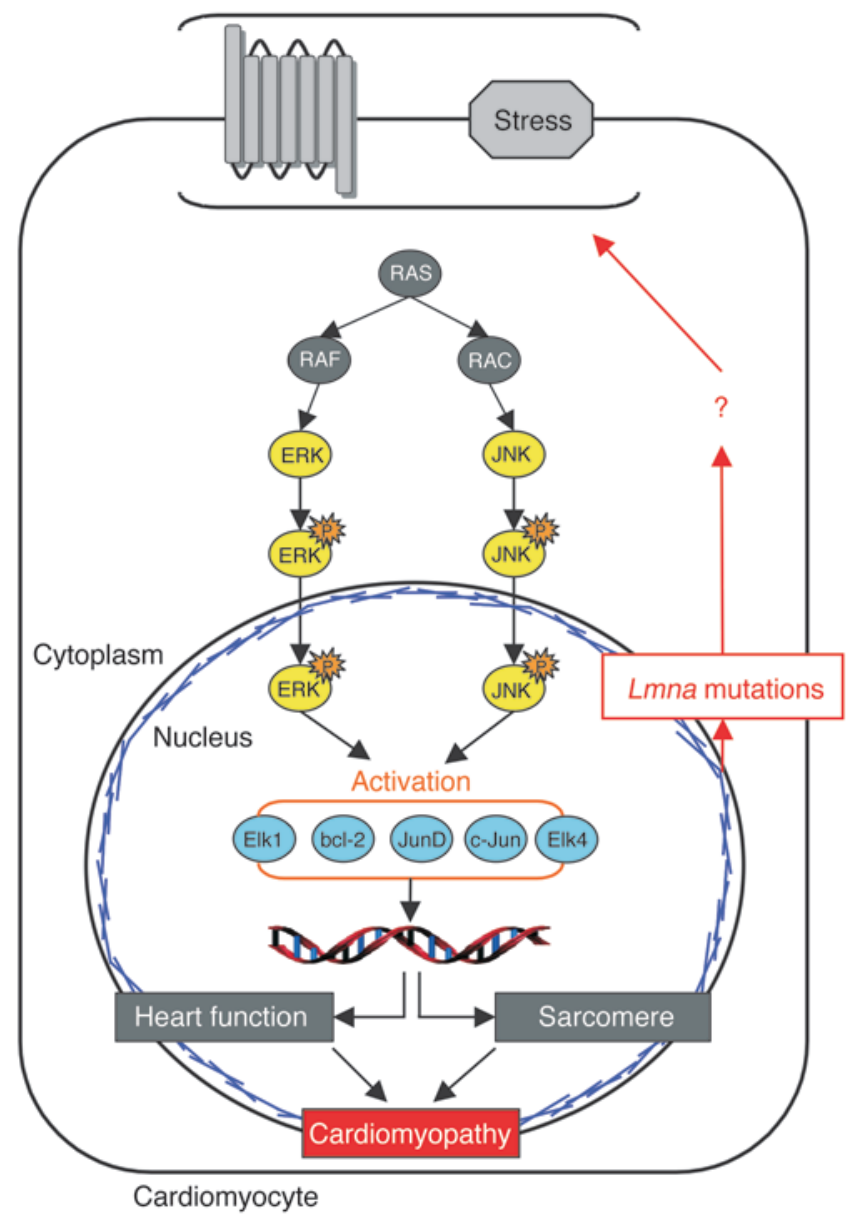


Cruz Biotechnology Inc.), and $\beta$-tubulin (Santa Cruz Biotechnology Inc.). Secondary Abs were HRP conjugated (Amersham Biosciences). Recognized proteins were visualized by enhanced chemiluminescence (ECL; Amersham Biosciences). Antibodies against $\beta$-tubulin and $\beta$-actin were used as internal controls to normalize the amounts of protein between immunoblots. Band densities were calculated using Scion Imaging software (version alpha 4.0.3.2; Scion Corp.) and normalized to the appropriate total extract to control for protein loading. Data are reported as mean \pm SD and are compared with respective controls using a 2-tailed Student's $t$ test.

Immunohistochemistry. Immunofluorescence staining for pERK1/2 was performed on frozen sections $(8 \mu \mathrm{m})$ of transversal cardiac muscles by fixing them in 3.7\% formaldehyde in PBS for 15 minutes, then blocking in 5\% fetal goat serum in PBS/triton for 1 hour. Cells were incubated in blocking solution with anti-pERK1/2 monoclonal antibody (Cell Signaling Technology) overnight at $4^{\circ} \mathrm{C}$ followed by PBS washing and incubation with Texas red-conjugated goat anti-mouse IgG secondary antibody (Invitrogen) and counterstained with $0.1 \mu \mathrm{g} / \mathrm{ml}$ DAPI (Sigma-Aldrich). Intensity of pERK1/2 in cardiocytes was measured using Scion Imaging software (version alpha 4.0.3.2; Scion Corp.). Data are reported as mean \pm SD and are compared with respective controls using a 2-tailed $t$ test.

Primary culture and isolation of ventricular myocytes. $L m n a^{+/+}$and $L m n a^{H 222 P / H 222 P}$ knockin mice (10 weeks of age) were anesthetized with pentofurane. Ventricular cardiomyocytes were isolated as described in the Alliance for Cellular Signaling procedure protocol PP00000125 (http://www.signaling-gateway.org/data/ProtocolLinks.html). For immunoblotting, cells were washed in $4^{\circ} \mathrm{C}$ PBS and lysed in extraction buffer. Lysates were centrifuged at $16,000 \mathrm{~g}$ and the supernatants collected. Immunoblotting was performed as described above.

Plasmid construction. To generate constructs to express lamins in transfected cells, cDNAs encoding wild-type Lamin A and Lamin A with H222P, N195K, R298C, R482W, N456I, and T528K aa substitutions were cloned in pegfp-C1 plasmid (Clontech) between $\mathrm{XhoI}$ and BamHI restriction endonuclease sites.

Cell culture and transfection to examine MAPK activation and localization. Cos-7 and C2C12 cells were maintained in Dulbecco's modified Eagle's medium supplemented with $10 \%$ fetal calf serum and $0.5 \%$ gentamycin at $37^{\circ} \mathrm{C}$ in a humidified atmosphere of $95 \%$ air and $5 \% \mathrm{CO}_{2}$. Cells were transfected with plasmids encoding GFP-wild-type Lamin A and GFP-H222P Lamin A using Lipofectamine 2000 (Invitrogen) according to the manufacturer's instructions. Cells were analyzed 48 hours after transfection. Cells were either fixed for 10 minutes in methanol at $-20^{\circ} \mathrm{C}$ or lysed in extraction buffer for subsequent immunoblotting.

Immunofluorescence microscopy. For immunofluorescence staining, fixed cells were incubated with rabbit Abs that recognize pERK1/2 or pJNK (Cell Signaling Technology). Cells were then washed and incubated with Texas red-conjugated goat anti-rabbit secondary Abs (Invitrogen). For immunohistochemistry, frozen sections $(8 \mu \mathrm{m})$ of transversal cardiac muscles were fixed in 3.7\% formaldehyde in PBS for 15 minutes and then blocked in 5\% fetal goat serum in PBS/Triton X-100 for 1 hour. Abs used for immunohistochemistry were primary rabbit anti-pERK1/2 (Cell Signaling Technology) and secondary Texas red-conjugated goat anti-rabbit (Invitrogen). Sections were counterstained with $0.1 \mu \mathrm{g} / \mathrm{ml}$ DAPI (SigmaAldrich). Immunofluorescence microscopy was performed on a Microphot SA (Nikon) microscope attached to a Spot RT slide camera (Diagnostic
Instruments). Images were processed using Adobe Photoshop 6.0 (Adobe Systems). Fluorescence intensity in cardiocytes was measured using Scion Imaging software (Scion Corp.). Data are reported as mean $\pm \mathrm{SD}$ and are compared with respective controls using a 2-tailed $t$ test.

Luciferase reportergene assays. Luciferase reporter assays for $c-J u n$ and Elk1 activation were carried out using PathDetect Trans-Reporting System (Stratagene). Cos-7 cells were plated in 12-well plates. The following day, cells were transfected with pegfp-N1 constructs encoding wild-type and mutant Lamin A proteins, pFA2-cJun or pFA2-Elk1, and pFR-Luc (Stratagene) using Lipofectamine 2000 (Invitrogen). To correct for transfection efficiency, a plasmid encoding $\beta$-gal (Promega) was cotransfected. After 24 hours, cells were trypsinized and protein lysates obtained and extracted according to the manufacturer's instructions (Promega). Luciferase activity was measured with a luminometer.

\section{Acknowledgments}

We thank A. Besana and P. McLaughlin for assistance isolating cardiomyocytes, X. Chien and A. Gharavi for assistance with realtime PCR, M. Thomas for assistance with the statistical analysis, V. Miljkovic for assistance with hybridization of Affymetrix GeneChips, R. Robinson for helpful discussions, and K. Schwartz and A.R. Marks for critically reviewing the manuscript. A. Muchir was supported in part by fellowship grants from Association Française contre les Myopathies and Fondation pour la Recherche Médicale. This work was supported by grants from the NIH (AR048997) and Muscular Dystrophy Association (MDA3711) to H.J. Worman; Human Frontier Science Program (RGP0057/2001-M) and Association Française contre les Myopathies (11057) to G. Bonne; and the Ministry of Education, Culture, Sports, and Technology, Japan, research grants from the Program for Promotion of Fundamental Studies in Health Sciences of the National Institute of Biomedical Innovation (NIBIO), Ichio Kanehara Foundation, and Association Française contre les Myopathies (11737) to T. Arimura.

Received for publication May 10, 2006, and accepted in revised form February 14, 2007.

Address correspondence to: Howard J. Worman, Department of Medicine, College of Physicians and Surgeons, Columbia University, 630 West 168th Street, P \& S Building, 10th floor, Room 509, New York, New York 10032, USA. Phone: (212) 305-8156; Fax: (212)305-6443; E-mail: hjw14@columbia.edu.

Paul Pavlidis's present address is: Department of Psychiatry, University of British Columbia, Vancouver, British Columbia, Canada.

Alan J. Herron's present address is: Center for Comparative Medicine and Department of Pathology, Baylor College of Medicine, Houston, Texas, USA.

Takuro Arimura's present address is: Department of Molecular Pathogenesis, Medical Research Institute, Tokyo Medical and Dental University, Tokyo, Japan.
1. Emery, A.E.H. 2000. Emery-Dreifuss muscular dystrophy - a 40 year retrospective. Neuromusc. Disord. 10:228-232.

2. Bione, S., et al. 1994. Identification of a novel $\mathrm{X}$-linked gene responsible for Emery-Dreifuss muscular dystrophy. Nat. Genet. 8:323-327.

3. Manilal, S., Nguyen, T.M., Sewry, C.A., and Morris, G.E. 1996. The Emery-Dreifuss muscular dystrophy protein, emerin, is a nuclear membrane protein. Hum. Mol. Genet. 5:801-808.

4. Nagano, A., et al. 1996. Emerin deficiency at the nuclear membrane in patients with Emery-Dreifuss muscular dystrophy. Nat. Genet. 12:254-259.

5. Bonne, G., et al. 1999. Mutations in the gene encoding lamin $\mathrm{A} / \mathrm{C}$ cause autosomal dominant Emery-Dreifuss muscular dystrophy. Nat. Genet.
21:285-288.

6. Raffaele Di Barletta, M., et al. 2000. Different mutations in the LMNA gene cause autosomal dominant and autosomal recessive Emery-Dreifuss muscular dystrophy. Am. J. Hum. Genet. 66:1407-1412.

7. Muchir, A., and Worman, H.J. 2004. The nuclear envelope and human disease. Physiology. 19:309-314.

8. Lin, F., and Worman, H.J. 1993. Structural organization 
of the human gene encoding nuclear lamin A and nuclear lamin C. J. Biol. Chem. 268:16321-16326.

9. Aebi, U., Cohn, J., Buhle, L., and Gerace, L. 1986. The nuclear lamina is a meshwork of intermediatetype filaments. Nature. 323:560-564.

10. Fisher, D.Z., Chaudhary, N., and Blobel, G. 1986. cDNA sequencing of nuclear lamins $A$ and $C$ reveals primary and secondary structural homology to intermediate filament proteins. Proc. Natl. Acad. Sci. U. S. A. 83:6450-6454.

11. Goldman, A.E., Maul, G., Steinert, P.M., Yang, H.Y., and Goldman, R.D. 1986. Keratin-like proteins that co-isolate with intermediate filaments of BHK- 21 cells are nuclear lamins. Proc. Natl. Acad. Sci. U. S. A. 83:3839-3843.

12. McKeon, F.D., Kirschner, M.W., and Caput, D. 1986. Homologies in both primary and secondary structure between nuclear envelope and intermediate filament proteins. Nature. 319:463-468.

13. Lammerding, J., et al. 2004. Lamin A/C deficiency causes defective nuclear mechanics and mechanotransduction. J. Clin. Invest. 113:370-378. doi:10.1172/JCI200419670.

14. Arimura, T., et al. 2005. Mouse model carrying H222P-Imna mutation develops muscular dystrophy and dilated cardiomyopathy similar to human striated muscle laminopathies. Hum. Mol. Genet. 14:155-169.

15. Hwang, J.J., et al. 2002. Microarray gene expression profiles in dilated and hypertrophic cardiomyopathic end-stage heart failure. Physiol. Genomics. 10:31-44.

16. Mukherjee, S., et al. 2003. Microarray analysis of changes in gene expression in a murine model of chronic chagasic cardiomyopathy. Parasitol. Res. 91:187-196.

17. Barrans, J.D., Allen, P.D., Stamatiou, D., Dzau, V.J., and Liew, C.C. 2002. Global gene expression profiling of end-stage dilated cardiomyopathy using a human cardiovascular-based cDNA microarray. Am. J. Pathol. 160:2035-2043.

18. Grzeskowiak, R., et al. 2003. Expression profiling of human idiopathic dilated cardiomyopathy. Cardiovasc. Res. 59:400-411.

19. Petrich, B.G., et al. 2002. c-Jun N-terminal kinase activation mediates downregulation of connexin 43 in cardiomyocytes. Circ. Res. 91:640-647.

20. Petrich, B.G., Molkentin, J.D., and Wang, Y. 2003. Temporal activation of c-Jun $\mathrm{N}$-terminal kinase in adult transgenic heart via cre-loxP-mediated DNA recombination. FASEB J. 17:749-751.

21. Chen, Z., et al. 2001. MAP kinases. Chem. Rev. 101:2449-2476.

22. Garrington, T.P., and Johnson, G.L. 1999. Organization and regulation of mitogen-activated protein kinase signaling pathways. Curr. Opin. Cell Biol. 11:211-218.

23. Baines, C.P., and Molkentin, J.D. 2005. STRESS signaling pathways that modulate cardiac myocyte apoptosis. J. Mol. Cell. Cardiol. 38:47-62.

24. Brunet, A., et al. 1999. Nuclear translocation of $\mathrm{p} 42 / \mathrm{p} 44$ mitogen-activated protein kinase is required for growth factor-induced gene expression and cell cycle entry. EMBOJ. 18:664-674

25. Hochholdinger, F., et al. 1999. Novel membranetargeted ERK1 and ERK2 chimeras which act as dominant negative, isotype-specific mitogen-activated protein kinase inhibitors of Ras-Raf-mediated transcriptional activation of c-fos in NIH $3 \mathrm{~T} 3$ cells. Mol. Cell. Biol. 19:8052-8065.

26. Qin, L.X., et al. 2006. Evaluation of methods for oligonucleoyides array data via quantitative real time PCR. BMC Bioinformatics. 7:23.

27. Millenaar, F.F., et al. 2006. How to decide? Different methods of calculating gene expression from short oligonucleotide array data will give different results. BMC Bioinformatics. 7:137.

28. Widmann, C., Gibson, S., Jarpe, M.B., and Johnson,
G.L. 1999. Mitogen-activated protein kinase: conservation of a three-kinase module from yeast to human. Physiol. Rev. 79:143-180.

29. Chi, H., et al. 2006. Dynamic regulation of proand anti-inflammatory cytokines by MAPK phosphatase 1 (MKP-1) in innate immune responses. Proc. Natl. Acad. Sci. U. S. A. 103:2274-2279.

30. Gillespie-Brown, J., Fuller, S.J., Bogoyevitch, M.A., Cowley, S., and Sugden, P.H. 1995. The mitogenactivated protein kinase kinase MEK1 stimulates a pattern of gene expression typical of the hypertrophic phenotype in rat ventricular cardiomyocytes. J. Biol. Chem. 270:28092-28096.

31. Thorburn, J., et al. 1995. Inhibition of a signaling pathway in cardiac muscle cells by active mitogen-activated protein kinase kinase. Mol. Biol. Cell. 6:1479-1490.

32. Braz, J.C., et al. 2003. Targeted inhibition of $p 38$ MAPK promotes hypertrophic cardiomyopathy through upregulation of calcineurin-NFAT signaling. J. Clin. Invest. 111:1475-1486. doi:10.1172/ JCI200317295

33. Nicol, R.L., et al. 2001. Activated MEK5 induces serial assembly of sarcomeres and eccentric cardiac hypertrophy. EMBO J. 20:2757-2767.

34. Cook, S.A., Sugden, P.H., and Clerk, A. 1999. Activation of c-Jun N-terminal kinases and p38mitogen-activated protein kinases in human heart failure secondary to ischaemic heart disease. J. Mol. Cell. Cardiol. 31:1429-1434.

35. Haq, S., et al. 2001. Differential activation of signal transduction pathways in human hearts with hypertrophy versus advanced heart failure. Circulation. 103:670-677.

36. Rodriguez-Viciana, P., et al. 2006. Germline mutations in genes within the MAPK pathway cause cardio-facio-cutaneous syndrome. Science. 311:1287-1290.

37. Bueno, O.F., et al. 2000. The MEK1-ERK1/2 signaling pathway promotes compensated cardiac hypertrophy in transgenic mice. EMBOJ. 19:6341-6350.

38. Woodman, S.E., et al. 2002. Caveolin-3 knock-out mice develop a progressive cardiomyopathy and show hyperactivation of the $\mathrm{p} 42 / 44$ MAPK cascade. J. Biol. Chem. 277:38988-38997.

39. Cohen, A.W., et al. 2003. Caveolin-1 null mice develop cardiac hypertrophy with hyperactivation of $\mathrm{p}$ 2/44 MAP kinase in cardiac fibroblasts. Am.J. Physiol. Cell Physiol. 284:C457-C474.

40. Luo, J., et al. 2005. Class IA phosphoinositide 3-kinase regulates heart size and physiological cardiac hypertrophy. Mol. Cell. Biol. 25:9491-9502.

41. Malarkey, K., et al. 1995. The regulation of tyrosine kinase signalling pathways by growth factor and Gprotein-coupled receptors. Biochem. J. 309:361-375.

42. Rosette, C., and Karin, M. 1996. Ultraviolet light and osmotic stress: activation of the JNK cascade through multiple growth factor and cytokine receptors. Science. 274:1194-1197.

43. Coleman, M.L., Densham, R.M., Croft, D.R., and Olson, M.F. 2006. Stability of p21 (Waf1/Cip1) $\mathrm{CDK}$ inhibitor protein is responsive to RhoA-mediated regulation of the actin cytoskeleton. Oncogene. 25:2708-2716

44. Smith, E.R., Smedberg, J.L., Rula, M.E., and Xu, X.X. 2004. Regulation of Ras-MAPK pathway mitogenic activity by restricting nuclear entry of activated MAPK in endoderm differentiation of embryonic carcinoma and stem cells. J. Cell Biol. 164:689-699.

45. Ivorra, C., et al. 2006. A mechanism of AP-1 suppression through interaction of $\mathrm{c}-\mathrm{Fos}$ with lamin A/C. Genes Dev. 20:307-320.

46. Schwartz, K., Boheler, K.R., de la Bastie, D., Lompre, A.M., and Mercadier, J.J. 1992. Switches in cardiac muscle gene expression as a result of pressure and volume overload. Am. J. Physiol. 262:R364-R369.

47. Chien, K.R., et al. 1993. Transcriptional regulation during cardiac growth and development. Annu. Rev.
Physiol. 55:77-95.

48. Hwang, J.J., et al. 2002. Microarray gene expression profiles in dilated and hypertrophic cardiomyopathic end-stage heart failure. Physiol. Genomics. 10:31-44.

49. Yung, C.K., Halperin, V.L., Tomaselli, G.F., and Winslow, R.L. 2004. Gene expression profiles in end-stage human idiopathic dilated cardiomyopathy: altered expression of apoptotic and cytoskeletal genes. Genomics. 83:281-297.

50. Swynghedauw, B. 1999. Molecular mechanisms of myocardial remodeling. Physiol. Rev. 79:215-262.

51. Swynghedauw, B., and Baillard, C. 2000. Biology of hypertensive cardiopathy. Curr. Opin. Cardiol. 15:247-253.

52. Yoshimine, K., et al. 1997. Altered expression of atrial natriuretic peptide and contractile protein genes in hypertrophied ventricle of JVS mice with systemic carnitine deficiency. J. Mol. Cell. Cardiol. 29:571-578.

53. Liao, P., et al. 2001. The in vivo role of p38 MAP kinases in cardiac remodeling and restrictive cardiomyopathy. Proc. Natl. Acad. Sci. U. S. A. 98:12283-12288.

54. Zheng, M., et al. 2004. Sarcoplasmic reticulum calcium defect in Ras-induced hypertrophic cardiomyopathy heart. Am. J. Physiol. Heart Circ. Physiol. 286:H424-H433.

55. Wang, J., et al. 2005. Targeted disruption of Smad4 in cardiomyocytes results in cardiac hypertrophy and heart failure. Circ. Res. 97:821-828.

56. Takahashi, T., Allen, P.D., and Izumo, S. 1992. Expression of A-, B-, and C-type natriuretic peptide genes in failing and developing human ventricles. Correlation with expression of the $\mathrm{Ca}(2+)$-ATPase gene. Circ. Res. 71:9-17.

57. Schwartz, K., et al. 1986. Alpha-skeletal muscle actin mRNA's accumulate in hypertrophied adult rat hearts. Circ. Res. 59:551-555.

58. Izumo, S., Nadal-Ginard, B., and Mahdavi, V. 1988. Protooncogene induction and reprogramming of cardiac gene expression produced by pressure overload. Proc. Natl. Acad. Sci. U. S. A. 85:339-343.

59. Ferrandi, C., et al. 2004. Inhibition of c-Jun N-terminal kinase decreases cardiomyocyte apoptosis and infarct size after myocardial ischemia and reperfusion in anaesthetized rats. Br. J. Pharmacol. 142:953-960.

60. Borsello, T., et al. 2003. A peptide inhibitor of c-Jun $\mathrm{N}$-terminal kinase protects against excitotoxicity and cerebral ischemia. Nat. Med. 9:1180-1186.

61. Wang, J., et al. 2003. A peptide inhibitor of c-Jun $\mathrm{N}$-terminal kinase protects against both aminoglycoside and acoustic trauma-induced auditory hair cell death and hearing loss. J. Neurosci. 23:8596-8607.

62. Minogue, A.M., et al. 2003. Activation of the c-Jun $\mathrm{N}$-terminal kinase signaling cascade mediates the effect of amyloid-beta on long term potentiation and cell death in hippocampus: a role for interleukin-1beta? J. Biol. Chem. 278:27971-27980.

63. Kuida, K., and Boucher, D.M. 2004. Functions of MAP kinases: insights from gene-targeting studies. J. Biochem. 135:653-656.

64. Mounkes, L.C., Kozlov, S.V., Rottman, J.N., and Stewart, C.L. 2005. Expression of an LMNA-N195K variant of A-type lamins results in cardiac conduction defects and death in mice. Hum. Mol. Genet. 14:2167-2180.

65. Pavlidis, P., Lewis, D.P., and Noble, W.S. 2002. Exploring gene expression data with class scores. Pac. Symp. Biocomput. 7:474-485.

66. Dennis, G., Jr., et al. 2003. DAVID: Database for Annotation, Visualization, and Discovery. Genome Biol. 4:P3.

67. Ponchel, F., et al. 2003. Real-time PCR based on SYBR-Green I fluorescence: an alternative to the TaqMan assay for a relative quantification of gene rearrangements, gene amplifications and micro gene deletions. BMC Biotechnol. 3:18. 